\title{
Crofton Measures and Minkowski Valuations
}

\author{
Franz E. Schuster
}

\begin{abstract}
A description of continuous rigid motion compatible Minkowski valuations is established. As an application we present a Brunn-Minkowski type inequality for intrinsic volumes of these valuations.
\end{abstract}

\section{Introduction}

As a generalization of the notion of measure, valuations on convex bodies (compact convex sets) have always played a central role in geometry. They were the critical ingredient in Dehn's solution of Hilbert's third problem and they have since been intimately tied to the dissection theory of polytopes. The starting point for many important new results in valuation theory is Hadwiger's 223] remarkable characterization of the intrinsic volumes as the continuous rigid motion invariant valuations. For more information on the history of valuations, see [27] and [41]. For some of the more recent results, see, e.g., [1-5, 12, 14, 20, 25, 28, 30, 33, 34].

In 2001, Alesker [2] has given a complete description of continuous translation invariant valuations on convex bodies thereby confirming, in a much stronger form, a conjecture by McMullen. Alesker's landmark result, now known as the Irreducibility Theorem, has subsequently led to the discovery of several new operations on the space of continuous translation invariant valuations, illuminating a new and rich algebraic structure $[2-5,14,15]$. In a different line of research, with a similar far reaching impact on the theory of valuations, Ludwig [29, 31, 32] first obtained characterizations of convex and star body valued valuations which are compatible with nondegenerate linear transformations, see also [22]. Her results revealed the underlying reason why such basic notions as projection, centroid and intersection bodies are indeed fundamental in the affine theory of convex bodies.

In this paper, we apply deep results on translation invariant real-valued valuations to establish a description of even and translation invariant convex body valued valuations which intertwine orthogonal transformations. Our result provides a significant extension of earlier work by Schneider [42], Kiderlen [24], and the author [47. As an application, we obtain a new Brunn-Minkowski type inequality for intrinsic volumes of these valuations, generalizing results by Lutwak [36] and the author [46]. 
Let $\mathcal{K}^{n}$ denote the space of convex bodies in $\mathbb{R}^{n}, n \geq 3$, endowed with the Hausdorff metric. A convex body $K$ is uniquely determined by its support function $h(K, u)=\max \{u \cdot x: x \in K\}$, for $u \in S^{n-1}$. For $i \in\{1, \ldots, n-1\}$, let $\mathrm{Gr}_{i, n}$ be the Grassmannian of $i$-dimensional subspaces in $\mathbb{R}^{n}$. The $i$ th projection function $\operatorname{vol}_{i}(K \mid \cdot)$ of $K \in \mathcal{K}^{n}$ is the continuous function on $\operatorname{Gr}_{i, n}$ defined such that $\operatorname{vol}_{i}(K \mid E)$, for $E \in \mathrm{Gr}_{i, n}$, is the $i$-dimensional volume of the orthogonal projection of $K$ onto $E$.

Definition $A \operatorname{map} \Phi: \mathcal{K}^{n} \rightarrow \mathcal{K}^{n}$ is called a Minkowski valuation if

$$
\Phi K+\Phi L=\Phi(K \cup L)+\Phi(K \cap L)
$$

whenever $K \cup L \in \mathcal{K}^{n}$ and addition on $\mathcal{K}^{n}$ is Minkowski addition.

Important examples of Minkowski valuations are such central notions as the projection and the difference operator: The projection body $\Pi K$ of $K$ is the convex body defined by

$$
h(\Pi K, u)=\operatorname{vol}_{n-1}\left(K \mid u^{\perp}\right), \quad u \in S^{n-1},
$$

where $u^{\perp}$ is the hyperplane orthogonal to $u$. The difference body $\mathrm{D} K$ of $K$ can be defined by

$$
h(\mathrm{D} K, u)=\operatorname{vol}_{1}(K \mid u), \quad u \in S^{n-1} .
$$

First results on a special class of Minkowski valuations were obtained by Schneider [42] in the 1970s, but only through the recent seminal work of Ludwig [29, 31] classifications of convex and star body valued valuations have become the focus of increased attention, see [21, 22, 24, 32, 44, 47. For example, Ludwig 31] established characterizations of the projection and the difference operator as unique Minkowski valuations which are compatible with affine transformations of $\mathbb{R}^{n}$.

In this article, we consider continuous translation invariant Minkowski valuations which are $\mathrm{O}(n)$ equivariant. This class of operators was studied under additional homogeneity assumptions first by Schneider [42], and more recently by Kiderlen [24] and the author [47]. A map $\Phi$ from $\mathcal{K}^{n}$ to $\mathcal{K}^{n}$ (or $\mathbb{R}$ ) is said to have degree $i$ if $\Phi(\lambda K)=\lambda^{i} \Phi K$ for $K \in \mathcal{K}^{n}$ and $\lambda \geq 0$. In the case of degree $i$ Minkowski valuations, Kiderlen [24, for $i=1$, and the author [47], for $i=n-1$, recently obtained representations of these maps by spherical convolution operators. A description of the intermediate degree cases $i \in\{2, \ldots, n-2\}$ remained open (by a result of McMullen [40], only integer degrees $0 \leq i \leq n$ can occur, cf. Section 3 ). 
As our main result we establish a representation for smooth translation invariant and $\mathrm{O}(n)$ equivariant Minkowski valuations $\Phi_{i}$ of degree $i \in\{1, \ldots, n-1\}$ which are even, i.e., $\Phi_{i}(-K)=\Phi_{i} K$ for $K \in \mathcal{K}^{n}$. We show that these maps are generated by convolution of the projection functions with (invariant) measures on the sphere.

Theorem 1.1 Let $\Phi_{i}: \mathcal{K}^{n} \rightarrow \mathcal{K}^{n}$ be a smooth translation invariant and $\mathrm{O}(n)$ equivariant Minkowski valuation of degree $i \in\{1, \ldots, n-1\}$. If $\Phi_{i}$ is even, then there exists an $\mathrm{O}(i) \times \mathrm{O}(n-i)$ invariant measure $\mu$ on $S^{n-1}$ such that for every $K \in \mathcal{K}^{n}$,

$$
h\left(\Phi_{i} K, \cdot\right)=\operatorname{vol}_{i}(K \mid \cdot) * \mu .
$$

Theorem 1.1 provides an extension of the previously known convolution representations of Kiderlen [24] and the author [47] (cf. Section 5). The convolution in (1.1) is induced from $\mathrm{O}(n)$ by identifying $S^{n-1}$ and $\mathrm{Gr}_{i, n}$ with the homogeneous spaces $\mathrm{O}(n) / \mathrm{O}(n-1)$ and $\mathrm{O}(n) / \mathrm{O}(i) \times \mathrm{O}(n-i)$. The generating measures for the projection and the difference operator are Dirac measures (cf. Section 4; for additional examples see Section 5).

The invariant signed measures in Theorem 1.1 are Crofton measures of associated real-valued valuations (cf. the proof of Theorem 1.1; for related results see [1] ). Additional properties of these measures and uniqueness will be discussed in Section 6.

The notion of smooth translation invariant real-valued valuations was introduced by Alesker in [3]. We will extend this definition to translation invariant Minkowski valuations which intertwine orthogonal transformations in Section 5.

As a consequence of Theorem 1.1, we obtain in Section 6 a stronger result, Theorem 6.3, describing the class of smooth translation invariant and $\mathrm{O}(n)$ equivariant even Minkowski valuations without additional assumption on the degree. We complement these results with the following:

Theorem 1.2 Every continuous translation invariant and $\mathrm{O}(n)$ equivariant even Minkowski valuation can be approximated uniformly on compact subsets of $\mathcal{K}^{n}$ by smooth translation invariant and $\mathrm{O}(n)$ equivariant even Minkowski valuations.

Consequently, the problem of describing continuous translation invariant and $\mathrm{O}(n)$ equivariant even Minkowski valuations is reduced to a description of smooth ones which is provided by Theorem 1.1 and Theorem 6.3 . 
For $i \in\{1, \ldots, n-1\}$, let $V_{i}(K)$ denote the $i$ th intrinsic volume of $K \in \mathcal{K}^{n}$ and denote by $\Pi_{i} K$ the projection body of order $i$ defined by

$$
h\left(\Pi_{i} K, u\right)=V_{i}\left(K \mid u^{\perp}\right), \quad u \in S^{n-1} .
$$

In [35, 36] Lutwak obtained an array of geometric inequalities for the intrinsic volumes of projection bodies which have been recently generalized in [46]. (For important recent related results on $L_{p}$ projection bodies, see [37-39]). A special case of [36. Theorem 6.2] is the following: If $K, L \in \mathcal{K}^{n}$ have non-empty interior and $i \in\{2, \ldots, n-1\}$, then

$$
V_{i+1}\left(\Pi_{i}(K+L)\right)^{1 / i(i+1)} \geq V_{i+1}\left(\Pi_{i} K\right)^{1 / i(i+1)}+V_{i+1}\left(\Pi_{i} L\right)^{1 / i(i+1)},
$$

with equality if and only if $K$ and $L$ are homothetic.

As an application of Theorem 1.1 we obtain a similar Brunn-Minkowski type inequality for all continuous translation invariant and $\mathrm{O}(n)$ equivariant even Minkowski valuations of a given degree.

Theorem 1.3 Let $\Phi_{i}: \mathcal{K}^{n} \rightarrow \mathcal{K}^{n}$ be a continuous translation invariant and $\mathrm{O}(n)$ equivariant even Minkowski valuation of degree $i \in\{2, \ldots, n-1\}$. If $K, L \in \mathcal{K}^{n}$ have non-empty interior, then

$$
V_{i+1}\left(\Phi_{i}(K+L)\right)^{1 / i(i+1)} \geq V_{i+1}\left(\Phi_{i} K\right)^{1 / i(i+1)}+V_{i+1}\left(\Phi_{i} L\right)^{1 / i(i+1)} .
$$

If $\Phi_{i}$ maps convex bodies with non-empty interiors to bodies with non-empty interiors, then equality holds if and only if $K$ and $L$ are homothetic.

Note that Theorem 1.3 provides a significant generalization of Lutwak's inequality (1.2) and the related results in [46]. We also remark that the classical Brunn-Minkowski inequalities for intrinsic volumes are special cases of Theorem 1.3 .

\section{Background material}

In the following we recall basic facts about convex bodies and mixed volumes. For quick reference, we state the geometric inequalities from the Brunn-Minkowski theory needed in the proof of Theorem 3. For general reference the reader may wish to consult the book by Schneider [43].

A convex body $K \in \mathcal{K}^{n}$ is uniquely determined by the values of its support function $h(K, \cdot)$ on $S^{n-1}$. From the definition of $h(K, \cdot)$, it is easily seen that $h(\vartheta K, u)=h\left(K, \vartheta^{-1} u\right)$ for every $u \in S^{n-1}$ and every $\vartheta \in \mathrm{O}(n)$. 
The Steiner point $s(K)$ of $K \in \mathcal{K}^{n}$ is the point in $K$ defined by

$$
s(K)=n \int_{S^{n-1}} h(K, u) u d u,
$$

where the integration is with respect to the rotation invariant probability measure on $S^{n-1}$.

For $K_{1}, K_{2} \in \mathcal{K}^{n}$ and $\lambda_{1}, \lambda_{2} \geq 0$, the support function of the Minkowski linear combination $\lambda_{1} K_{1}+\lambda_{2} K_{2}$ is

$$
h\left(\lambda_{1} K_{1}+\lambda_{2} K_{2}, \cdot\right)=\lambda_{1} h\left(K_{1}, \cdot\right)+\lambda_{2} h\left(K_{2}, \cdot\right) .
$$

By a theorem of Minkowski, the volume of a Minkowski linear combination $\lambda_{1} K_{1}+\ldots+\lambda_{m} K_{m}$ of convex bodies $K_{1}, \ldots, K_{m}$ can be expressed as a homogeneous polynomial of degree $n$ :

$$
V\left(\lambda_{1} K_{1}+\ldots+\lambda_{m} K_{m}\right)=\sum_{i_{1}, \ldots, i_{n}} V\left(K_{i_{1}}, \ldots, K_{i_{n}}\right) \lambda_{i_{1}} \cdots \lambda_{i_{n}} .
$$

The coefficients $V\left(K_{i_{1}}, \ldots, K_{i_{n}}\right)$ are called mixed volumes of $K_{i_{1}}, \ldots, K_{i_{n}}$. These functionals are nonnegative, symmetric and translation invariant. Clearly, their diagonal form is ordinary volume, i.e., $V(K, \ldots, K)=V(K)$.

For $K, L \in \mathcal{K}^{n}$ and $0 \leq i \leq n-1$, we write $W_{i}(K, L)$ to denote the mixed volume $V(K, \ldots, K, B, \ldots, B, L)$, where $K$ appears $n-i-1$ times and the Euclidean unit ball $B$ appears $i$ times. The mixed volume $W_{i}(K, K)$ will be written as $W_{i}(K)$ and is called the ith quermassintegral of $K$. The $i$ th intrinsic volume $V_{i}(K)$ of $K$ is defined by

$$
\kappa_{n-i} V_{i}(K)=\left(\begin{array}{c}
n \\
i
\end{array}\right) W_{n-i}(K),
$$

where $\kappa_{n}$ is the $n$-dimensional volume of the Euclidean unit ball in $\mathbb{R}^{n}$. A special case of (2.1) is the classical Steiner formula for the volume of the outer parallel body of $K$ at distance $\varepsilon>0$ :

$$
V(K+\varepsilon B)=\sum_{i=0}^{n} \varepsilon^{i}\left(\begin{array}{c}
n \\
i
\end{array}\right) W_{i}(K)=\sum_{i=0}^{n} \varepsilon^{n-i} \kappa_{n-i} V_{i}(K) .
$$

For $1 \leq i \leq n-1$, the quermassintegral $W_{n-i}(K)$ of $K \in \mathcal{K}^{n}$ can also be defined by

$$
W_{n-i}(K)=\frac{\kappa_{n}}{\kappa_{i}} \int_{\operatorname{Gr}_{i, n}} \operatorname{vol}_{i}(K \mid E) d E,
$$

where the integration is with respect to the rotation invariant probability measure on $\mathrm{Gr}_{i, n}$. 
Let $\mathcal{K}_{\mathrm{o}}^{n}$ denote the set of convex bodies in $\mathbb{R}^{n}$ with non-empty interior. One of the fundamental inequalities for mixed volumes is the general Minkowski inequality: If $K, L \in \mathcal{K}_{\mathrm{o}}^{n}$ and $0 \leq i \leq n-2$, then

$$
W_{i}(K, L)^{n-i} \geq W_{i}(K)^{n-i-1} W_{i}(L),
$$

with equality if and only if $K$ and $L$ are homothetic.

A consequence of the Minkowski inequality (2.4) is the Brunn-Minkowski inequality for quermassintegrals: If $K, L \in \mathcal{K}_{\mathrm{o}}^{n}$ and $0 \leq i \leq n-2$, then

$$
W_{i}(K+L)^{1 /(n-i)} \geq W_{i}(K)^{1 /(n-i)}+W_{i}(L)^{1 /(n-i)},
$$

with equality if and only if $K$ and $L$ are homothetic.

For $K, K_{1}, \ldots, K_{i} \in \mathcal{K}^{n}$ and $\mathbf{C}=\left(K_{1}, \ldots, K_{i}\right)$, let $V_{i}(K, \mathbf{C})$ denote the mixed volume $V\left(K, \ldots, K, K_{1}, \ldots, K_{i}\right)$ with $n-i$ copies of $K$. A further generalization of inequality (2.5) (but without equality conditions) is the following: If $0 \leq i \leq n-2, K, L, K_{1}, \ldots, K_{i} \in \mathcal{K}^{n}$ and $\mathbf{C}=\left(K_{1}, \ldots, K_{i}\right)$, then

$$
V_{i}(K+L, \mathbf{C})^{1 /(n-i)} \geq V_{i}(K, \mathbf{C})^{1 /(n-i)}+V_{i}(L, \mathbf{C})^{1 /(n-i)} .
$$

A convex body $K \in \mathcal{K}_{\mathrm{o}}^{n}$ is also determined up to translation by its surface area measure $S_{n-1}(K, \cdot)$. Recall that for a Borel set $\omega \subseteq S^{n-1}, S_{n-1}(K, \omega)$ is the $(n-1)$-dimensional Hausdorff measure of the set of all boundary points of $K$ at which there exists a normal vector of $K$ belonging to $\omega$. The relation $S_{n-1}(\lambda K, \cdot)=\lambda^{n-1} S_{n-1}(K, \cdot)$ holds for all $K \in \mathcal{K}^{n}$ and every $\lambda \geq 0$. For $\vartheta \in \mathrm{O}(n)$, we have $S_{n-1}(\vartheta K, \cdot)=\vartheta S_{n-1}(K, \cdot)$, where $\vartheta S_{n-1}(K, \cdot)$ is the image measure of $S_{n-1}(K, \cdot)$ under $\vartheta \in \mathrm{O}(n)$.

The surface area measure $S_{n-1}(K, \cdot)$ of $K \in \mathcal{K}^{n}$ satisfies the Steiner-type formula

$$
S_{n-1}(K+\varepsilon B, \cdot)=\sum_{i=0}^{n-1} \varepsilon^{n-1-i}\left(\begin{array}{c}
n-1 \\
i
\end{array}\right) S_{i}(K, \cdot) .
$$

The measure $S_{i}(K, \cdot)$ is called the area measure of order $i$ of $K \in \mathcal{K}^{n}$.

We conclude this section with an integral representation connecting area measures and quermassintegrals: If $K, L \in \mathcal{K}^{n}$ and $0 \leq i \leq n-1$, then

$$
W_{n-1-i}(K, L)=\frac{1}{n} \int_{S^{n-1}} h(L, u) d S_{i}(K, u) .
$$




\section{Translation invariant valuations}

In this section, we collect some results from the theory of real-valued translation invariant valuations. In particular, we recall the definition of smooth valuations and the notion of Crofton measures.

A function $\phi: \mathcal{K}^{n} \rightarrow \mathcal{A}$ into an abelian semigroup $(\mathcal{A},+)$ is called a valuation if

$$
\phi(K \cup L)+\phi(K \cap L)=\phi(K)+\phi(L),
$$

whenever $K, L, K \cup L \in \mathcal{K}^{n}$. The notion of valuation as defined here is a classical concept from convex geometry. However, we remark that Alesker has recently introduced a broader notion of valuation in the more general setting of smooth manifolds, see [6-8, 10, 13].

A valuation $\phi$ is called translation invariant if $\phi(K+x)=\phi(K)$ for all $x \in \mathbb{R}^{n}$ and $K \in \mathcal{K}^{n}$. We denote the vector space of continuous translation invariant real-valued valuations by $\mathbf{V a l}$ and we write $\mathbf{V a l}_{i}$ for its subspace of all valuations of degree $i$. A valuation $\phi \in \mathbf{V a l}$ is said to be even (resp. odd) if $\phi(-K)=(-1)^{\alpha} \phi(K)$ with $\alpha=0$ (resp. $\alpha=1$ ) for all $K \in \mathcal{K}^{n}$. We write $\mathrm{Val}_{i}^{+} \subseteq \mathrm{Val}_{i}$ for the subspace of even valuations of degree $i$ and $\mathrm{Val}_{i}^{-}$ to denote the space of odd valuations of degree $i$, respectively.

The following result was obtained by McMullen:

Theorem 3.1 (McMullen [40])

$$
\mathbf{V a l}=\bigoplus_{i=0}^{n} \mathbf{V a l}_{i}^{+} \oplus \mathbf{V a l}_{i}^{-} .
$$

It follows from Theorem 3.1 that the space Val becomes a Banach space under the norm

$$
\|\phi\|=\sup \{|\phi(K)|: K \subseteq B\}
$$

\section{Examples:}

(a) It is easy to see that the space $\mathbf{V a l}_{0}$ is one-dimensional and is spanned by the Euler characteristic $\chi$ (recall that $\chi(K)=1$ for all $\left.K \in \mathcal{K}^{n}\right)$.

(b) Hadwiger [23, p. 79] has shown that $\mathbf{V a l}_{n}$ is also one-dimensional and is spanned by ordinary volume $V$.

(c) For $i \in\{0, \ldots, n\}$, let us fix convex bodies $\mathbf{C}=\left(L_{1}, \ldots, L_{i}\right)$. The mixed volume $V_{i}(K, \mathbf{C})$ belongs to $\mathbf{V a l}_{n-i}$. 
The group $\mathrm{GL}(n)$ has a natural continuous representation $\rho$ on the Banach space Val: For every $A \in \mathrm{GL}(n)$ and $K \in \mathcal{K}^{n}$,

$$
(\rho(A) \phi)(K)=\phi\left(A^{-1} K\right), \quad \phi \in \text { Val. }
$$

Note that the subspaces $\mathbf{V a l}_{i}^{ \pm} \subseteq \mathbf{V a l}$ are invariant under this $\mathrm{GL}(n)$ action. The Irreducibility Theorem of Alesker states the following:

Theorem 3.2 (Alesker [2]) The natural representation of $\mathrm{GL}(n)$ on $\mathrm{Val}_{i}^{ \pm}$ is irreducible for any $i \in\{0, \ldots, n\}$.

The Irreducibility Theorem directly implies a conjecture by McMullen that the linear combinations of mixed volumes are dense in Val (see [2]).

In the following we will further illustrate the strength of the Irreducibility Theorem by constructing an alternative description of translation invariant even valuations: Assume that $1 \leq i \leq n-1$. For any finite Borel measure $\mu$ on $\mathrm{Gr}_{i, n}$ define an even valuation $\mathrm{A}_{i} \mu \in \mathbf{V a l}_{i}^{+}$by

$$
\left(\mathrm{A}_{i} \mu\right)(K)=\int_{\mathrm{Gr}_{i, n}} \operatorname{vol}_{i}(K \mid E) d \mu(E)
$$

Clearly, the image of the map $\mathrm{A}_{i}$ is a $\mathrm{GL}(n)$ invariant subspace of $\mathrm{Val}_{i}^{+}$. Therefore, by Theorem 3.2, this image is dense in $\mathbf{V a l}_{i}^{+}$.

Definition A finite Borel measure $\mu$ on $\mathrm{Gr}_{i, n}, 1 \leq i \leq n-1$, is called a Crofton measure for the valuation $\phi \in \mathbf{V a l}_{i}^{+}$if $\mathrm{A}_{i} \mu=\phi$.

The classical Crofton formula is a result from the early days of integral geometry relating the length of a curve in the plane to the expected number of intersection points with random lines. Higher-dimensional generalizations have become known as linear kinematic formulas. For more information on Crofton formulas the reader is referred to the recent book by Schneider and Weil [45]. Further details on Crofton measures of valuations (not necessarily translation invariant) can be found in [11].

In the following it will be important for us to work with a subset of valuations in $\mathbf{V a l}_{i}^{+}$which admit a Crofton formula (3.1).

Definition $A$ valuation $\phi \in \mathbf{V a l}$ is called smooth if the map $\mathrm{GL}(n) \rightarrow$ Val defined by $A \mapsto \rho(A) \phi$ is infinitely differentiable.

The notion of smooth valuations is a special case of the more general concept of smooth vectors in a representation space (see, e.g., [50, p. 31]). 
We denote the space of smooth translation invariant valuations by $\mathbf{V a l}^{\infty}$ and we write $\mathbf{V a l}_{i}^{ \pm, \infty}$ for the subspace of smooth valuations in $\mathbf{V a l}_{i}^{ \pm}$. From representation theory it is well known (cf. [50, p. 32]) that the set of smooth valuations $\mathbf{V a l}_{i}^{ \pm, \infty}$ is a dense $\operatorname{GL}(n)$ invariant subspace of $\mathbf{V a l}_{i}^{ \pm}$and one easily deduces the following decomposition:

$$
\mathbf{V a l}^{\infty}=\bigoplus_{i=0}^{n} \mathbf{V a l}_{i}^{+, \infty} \oplus \mathbf{V a l}_{i}^{-, \infty}
$$

Now consider the restriction of the map $\mathrm{A}_{i}, 1 \leq i \leq n-1$, defined in (3.1) to smooth functions:

$$
\left(\mathrm{A}_{i} f\right)(K)=\int_{\mathrm{Gr}_{i, n}} \operatorname{vol}_{i}(K \mid E) f(E) d E, \quad f \in C^{\infty}\left(\operatorname{Gr}_{i, n}\right) .
$$

Clearly, the valuation $\mathrm{A}_{i} f$ is smooth, i.e., $\mathrm{A}_{i} f \in \mathbf{V a l}_{i}^{+, \infty}$. Moreover, it follows from a deep result of Alesker and Bernstein [9] that any smooth translation invariant and even valuation admits such a Crofton formula. In order to explain this fact we need the cosine transform on Grassmannians.

Assume that $1 \leq i \leq n-1$. For two subspaces $E, F \in \mathrm{Gr}_{i, n}$, the cosine of the angle between $E$ and $F$ is defined by

$$
|\cos (E, F)|=\operatorname{vol}_{i}\left(\operatorname{Pr}_{F}(M)\right)
$$

where $M$ is any subset of $E$ with $\operatorname{vol}_{i}(M)=1$ and $\operatorname{Pr}_{F}$ denotes the orthogonal projection onto $F$. (This definition does not depend on the choice of $M \subseteq E$.) The cosine transform $\mathrm{C}_{i}: C\left(\mathrm{Gr}_{i, n}\right) \rightarrow C\left(\mathrm{Gr}_{i, n}\right)$ is defined by

$$
\left(\mathrm{C}_{i} f\right)(F)=\int_{\mathrm{Gr}_{i, n}}|\cos (E, F)| f(E) d E .
$$

Alesker and Bernstein established a fundamental connection between the range of the cosine transform and even translation invariant valuations. This result is based on an imbedding $\mathrm{K}_{i}: \mathrm{Val}_{i}^{+} \rightarrow C\left(\mathrm{Gr}_{i, n}\right)$ due to Klain: For $\phi \in \mathrm{Val}_{i}^{+}$and every $E \in \mathrm{Gr}_{i, n}$, consider the restriction $\phi_{E}$ of $\phi$ to convex bodies in $E$. This is a continuous translation invariant valuation of degree $i$ in $E$. Hence, by a result of Hadwiger [23, p. 79], $\phi_{E}=g(E) \operatorname{vol}_{i}$, where $g(E)$ is a constant depending on $E$. The map $\mathrm{K}_{i}: \mathrm{Val}_{i}^{+} \rightarrow C\left(\mathrm{Gr}_{i, n}\right)$, defined by $\mathrm{K}_{i} \phi=g$, turns out to be injective by a result of Klain [26]. The function $g$ is called the Klain function of the valuation $\phi$. 
The Alesker-Bernstein theorem can be stated as follows:

Theorem 3.3 (Alesker and Bernstein [9]) Suppose that $1 \leq i \leq n-1$. The image of the Klain imbedding $\mathrm{K}_{i}: \mathbf{V a l}_{i}^{+, \infty} \rightarrow C^{\infty}\left(\mathrm{Gr}_{i, n}\right)$ coincides with the image of the cosine transform $\mathrm{C}_{i}: C^{\infty}\left(\mathrm{Gr}_{i, n}\right) \rightarrow C^{\infty}\left(\mathrm{Gr}_{i, n}\right)$.

We remark that this version of the Alesker-Bernstein theorem is obtained from the main results in [9] by an application of the Casselman-Wallach theorem [16] (cf. [3, p. 72]).

For $1 \leq i \leq n-1$, let $\mathbf{T}_{i}^{\infty}$ denote the image of smooth functions on $\mathrm{Gr}_{i, n}$ under the cosine transform $\mathrm{C}_{i}$. It is well known that $\mathrm{C}_{i}$ is not injective for $2 \leq i \leq n-2$. However, since $\mathrm{C}_{i}$ is selfadjoint its restriction to $\mathbf{T}_{i}^{\infty}$ has trivial kernel. Moreover, from an application of the Casselman-Wallach theorem [16] to the main result of [9], Alesker [3, p. 73] deduced that

$$
\mathrm{C}_{i}\left(\mathbf{T}_{i}^{\infty}\right)=\mathbf{T}_{i}^{\infty} .
$$

Now suppose that $F \in \mathrm{Gr}_{i, n}$. Then, for any $f \in C\left(\mathrm{Gr}_{i, n}\right)$ and any convex body $K \subseteq F$,

$$
\left(\mathrm{A}_{i} f\right)(K)=\operatorname{vol}_{i}(K) \int_{\mathrm{Gr}_{i, n}}|\cos (E, F)| f(E) d E .
$$

Consequently, the Klain function of the valuation $\mathrm{A}_{i} f$ is equal to the cosine transform $\mathrm{C}_{i} f$ of $f$. Thus, we obtain from Theorem 3.3 and (3.3):

Corollary 3.4 For any valuation $\phi \in \mathrm{Val}_{i}^{+, \infty}$, there exists a unique smooth measure $\mu \in \mathbf{T}_{i}^{\infty}$ such that $\mu$ is a Crofton measure for $\phi$.

We conclude this section with a commutative diagram for the crucial isomorphisms needed in the following:

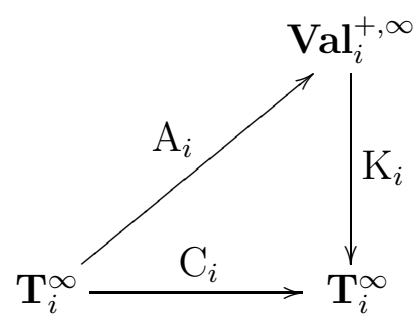




\section{Convolutions}

Here we recall the basic notion of convolution on the compact Lie group $\mathrm{O}(n)$ and the homogeneous spaces $\mathrm{O}(n) / \mathrm{O}(n-1)$ and $\mathrm{O}(n) / \mathrm{O}(i) \times \mathrm{O}(n-i)$. At the end of this section, we establish an auxiliary result which is critical in the proof of Theorem 1.3. As a general reference for this section we recommend the article by Grinberg and Zhang [19].

Let $C(\mathrm{O}(n))$ denote the space of continuous functions on $\mathrm{O}(n)$ with the uniform topology. In this article all measures are signed finite Borel measures. For $f \in C(\mathrm{O}(n))$ and a measure $\mu$ on $\mathrm{O}(n)$, the canonical pairing is

$$
\langle\mu, f\rangle=\langle f, \mu\rangle=\int_{\mathrm{O}(n)} f(\vartheta) d \mu(\vartheta) .
$$

We will frequently identify a continuous function $f \in C(\mathrm{O}(n))$ with the absolutely continuous measure (with respect to Haar probability measure on $\mathrm{O}(n))$ with density $f$. The canonical pairing is then consistent with the usual inner product on $C(\mathrm{O}(n))$.

For $\vartheta \in \mathrm{O}(n)$, the left translation $\vartheta f$ of $f \in C(\mathrm{O}(n))$ is defined by

$$
\vartheta f(\eta)=f\left(\vartheta^{-1} \eta\right)
$$

For a measure $\mu$ on $\mathrm{O}(n)$, we set

$$
\langle\vartheta \mu, f\rangle=\left\langle\mu, \vartheta^{-1} f\right\rangle, \quad f \in C(\mathrm{O}(n)) .
$$

Then $\vartheta \mu$ is just the image measure of $\mu$ under the rotation $\vartheta$.

For $f \in C(\mathrm{O}(n))$, the function $\widehat{f} \in C(\mathrm{O}(n))$ is defined by

$$
\widehat{f}(\vartheta)=f\left(\vartheta^{-1}\right) .
$$

For a measure $\mu$ on $\mathrm{O}(n)$, we define the measure $\widehat{\mu}$ by

$$
\langle\widehat{\mu}, f\rangle=\langle\mu, \widehat{f}\rangle, \quad f \in C(\mathrm{O}(n)) .
$$

For $f, g \in C(\mathrm{O}(n))$, the convolution $f * g \in C(\mathrm{O}(n))$ is defined by

$$
(f * g)(\eta)=\int_{\mathrm{O}(n)} f\left(\eta \vartheta^{-1}\right) g(\vartheta) d \vartheta=\int_{\mathrm{O}(n)} f(\vartheta) g\left(\vartheta^{-1} \eta\right) d \vartheta
$$

where integration is with respect to the Haar probability measure on $\mathrm{O}(n)$. 
For a measure $\mu$ on $\mathrm{O}(n)$ and a function $f \in C(\mathrm{O}(n))$, the convolutions $\mu * f \in C(\mathrm{O}(n))$ and $f * \mu \in C(\mathrm{O}(n))$ are defined by

$$
(f * \mu)(\eta)=\int_{\mathrm{O}(n)} f\left(\eta \vartheta^{-1}\right) d \mu(\vartheta), \quad(\mu * f)(\eta)=\int_{\mathrm{O}(n)} \vartheta f(\eta) d \mu(\vartheta) .
$$

From this definition, it follows that $f * \mu$ and $\mu * f$ are $C^{\infty}$ if $f \in C^{\infty}(\mathrm{O}(n))$.

We emphasize that, if $\mu$ is a measure on $\mathrm{O}(n)$, then, by (4.1), for all $f \in C(\mathrm{O}(n))$ and every $\vartheta \in \mathrm{O}(n)$,

$$
(\vartheta f) * \mu=\vartheta(f * \mu) .
$$

Thus, the convolution from the right gives rise to operators on $C(\mathrm{O}(n))$ which intertwine orthogonal transformations.

Using (4.1), it is also easy to verify that for $f, g \in C(\mathrm{O}(n))$ and a measure $\sigma$ on $\mathrm{O}(n)$,

$$
\langle g * \sigma, f\rangle=\langle g, f * \widehat{\sigma}\rangle .
$$

This identity allows us to define the convolution $\mu * \sigma$ of two measures $\mu, \sigma$ on $\mathrm{O}(n)$ by

$$
\langle\mu * \sigma, f\rangle=\langle\sigma, \widehat{\mu} * f\rangle=\langle\mu, f * \widehat{\sigma}\rangle, \quad f \in C(\mathrm{O}(n)) .
$$

It is easy to check that the convolution of functions and measures on $\mathrm{O}(n)$ defined in this way is associative. However, the convolution is in general not commutative. If $\mu, \sigma$ are measures on $\mathrm{O}(n)$, then

$$
\widehat{\mu * \sigma}=\widehat{\sigma} * \widehat{\mu} \text {. }
$$

For the following Lemma see [19, p. 85].

Lemma 4.1 Let $\mu, \mu_{m}, m \in \mathbb{N}$, be measures on $\mathrm{O}(n)$ and let $f \in C(\mathrm{O}(n))$. If $\mu_{m} \rightarrow \mu$ weakly, then $f * \mu_{m} \rightarrow f * \mu$ and $\mu_{m} * f \rightarrow \mu * f$ uniformly.

In the following we extend the definition of convolutions to functions and measures on the homogeneous spaces

$$
S^{n-1}=\mathrm{O}(n) / \mathrm{O}(n-1) \quad \text { and } \quad \mathrm{Gr}_{i, n}=\mathrm{O}(n) / \mathrm{O}(i) \times \mathrm{O}(n-i) .
$$

In order to treat both cases simultaneously let $H$ denote a closed subgroup of $\mathrm{O}(n)$. We consider the compact homogeneous space $\mathrm{O}(n) / H$.

Let $\pi: \mathrm{O}(n) \rightarrow \mathrm{O}(n) / H$ be the canonical projection and write $\pi(\vartheta)=\bar{\vartheta}$. If $e \in \mathrm{O}(n)$ denotes the identity map, then $H$ is the stabilizer in $\mathrm{O}(n)$ of $\bar{e} \in \mathrm{O}(n) / H$ and we have $\bar{\vartheta}=\vartheta \bar{e}$ for every $\vartheta \in \mathrm{O}(n)$. 
Every continuous function $f$ on $\mathrm{O}(n) / H$ gives rise to a continuous right $H$-invariant function $\breve{f}=f \circ \pi$ on $\mathrm{O}(n)$. Conversely, every $f \in C(\mathrm{O}(n))$ induces a continuous function $\bar{f}$ on $\mathrm{O}(n) / H$, defined by

$$
\bar{f}(\bar{\eta})=\int_{H} f(\eta \vartheta) d \vartheta .
$$

If $f \in C(\mathrm{O}(n))$ is right $H$-invariant, then $f=\bar{f} \circ \pi$. Therefore, the subspace of right $H$-invariant functions in $C(\mathrm{O}(n))$ is isomorphic to $C(\mathrm{O}(n) / H)$.

For a measure $\mu$ on $\mathrm{O}(n) / H$, we define the measure $\breve{\mu}$ on $\mathrm{O}(n)$ by

$$
\langle\breve{\mu}, f\rangle=\langle\mu, \bar{f}\rangle .
$$

In this way we also obtain a one-to-one correspondence between measures on $\mathrm{O}(n) / H$ and right $H$-invariant measures on $\mathrm{O}(n)$.

The convolutions of functions and measures on $\mathrm{O}(n) / H$ can be defined via their identification with right $H$-invariant functions and measures on $\mathrm{O}(n)$. For example, the convolution $f * \mu \in C(\mathrm{O}(n) / H)$ of $f \in C(\mathrm{O}(n) / H)$ with a measure $\mu$ on $\mathrm{O}(n) / H$ is defined by

$$
(f * \mu)(\bar{\eta})=(\breve{f} * \breve{\mu})(\eta)=\int_{\mathrm{O}(n)} f\left(\eta \vartheta^{-1} \bar{e}\right) d \breve{\mu}(\vartheta) .
$$

In the same way we can define convolutions between different homogeneous spaces: Let $H_{1}, H_{2}$ be two closed subgroups of $\mathrm{O}(n)$. If $f \in C\left(\mathrm{O}(n) / H_{1}\right)$ and $g \in C\left(\mathrm{O}(n) / H_{2}\right)$, then $\breve{f} * \breve{g}$ defines a continuous right $H_{2}$-invariant function on $\mathrm{O}(n)$ and thus can be identified with a continuous function on $\mathrm{O}(n) / H_{2}$.

It follows from (4.5) that the Dirac measure $\delta_{\bar{e}}$ on $\mathrm{O}(n) / H$ is the unique rightneutral element for the convolution on $\mathrm{O}(n) / H$. If $f \in C(\mathrm{O}(n))$, then

$$
f * \delta_{\bar{e}}=\bar{f}
$$

is right $H$-invariant and

$$
\delta_{\bar{e}} * f=\int_{H} \vartheta f d \vartheta
$$

defines a left $H$-invariant function on $\mathrm{O}(n)$.

An essential role among functions (and measures) on $\mathrm{O}(n)$ play the $H$-biinvariant functions. They can be identified with functions on $\mathrm{O}(n) / H$ with the property that $\vartheta f=f$ for every $\vartheta \in H$. We call a $H$-invariant function on $\mathrm{O}(n) / H$ zonal. If $f, g \in C(\mathrm{O}(n) / H)$, then, by (4.6) and (4.7),

$$
f * g=\left(f * \delta_{\bar{e}}\right) * g=f *\left(\delta_{\bar{e}} * g\right) .
$$


Consequently, for convolutions from the right on $\mathrm{O}(n) / H$, it is sufficient to consider zonal functions and measures.

If $f \in C(\mathrm{O}(n))$ is $H$-biinvariant (or, equivalently, $f \in C(\mathrm{O}(n) / H)$ is zonal), then the function $\widehat{f}$ is also $H$-biinvariant and thus can be identified with a zonal function on $\mathrm{O}(n) / H$.

It is trivial to verify that if $f \in C\left(S^{n-1}\right)$ is zonal, then $\widehat{f}=f$. The corresponding result for the Grassmannian will be crucial in the proof of Theorem 1.3 .

Lemma 4.2 If $f \in C\left(\mathrm{Gr}_{i, n}\right)$ is $\mathrm{O}(i) \times \mathrm{O}(n-i)$ invariant, then

$$
\widehat{f}=f \text {. }
$$

Proof. Let $H$ denote the subgroup $\mathrm{O}(i) \times \mathrm{O}(n-i)$. We identify $f$ with a $H$-biinvariant function on $\mathrm{O}(n)$. We will show that for any $\vartheta \in \mathrm{O}(n)$,

$$
H \vartheta^{-1} H=H \vartheta H \text {. }
$$

Clearly, (4.9) implies that $\widehat{f}=f$.

For the following short proof of (4.9) the author is obliged to S. Alesker. Replacing $i$ with $n-i$ if necessary, we may assume that $i \leq n / 2$. Fix an orthonormal basis $\left\{b_{1}, \ldots, b_{n}\right\}$ of $\mathbb{R}^{n}$ and let $I_{n}$ denote the identity matrix. Consider the torus $T \subseteq \mathrm{O}(n)$ consisting of rotations of the form

$$
\left(\begin{array}{ccc|ccc|c}
\cos \alpha_{1} & & & -\sin \alpha_{1} & & \\
& \ddots & & & \ddots & \\
& & \cos \alpha_{i} & & & -\sin \alpha_{i} & \\
\hline \sin \alpha_{1} & & & \cos \alpha_{1} & & \\
& \ddots & & & \ddots & & \\
& & \sin \alpha_{i} & & & \cos \alpha_{i} & \\
\hline & & & I_{n-2 i}
\end{array}\right)
$$

It is well known (and can be generalized appropriately to the setting of Riemannian symmetric pairs (see, e.g., [49, Chapter II])) that

$$
\mathrm{O}(n)=H T H \text {. }
$$

Consequently, we can assume for the proof of (4.9) that $\vartheta \in T$. But now it is straightforward to verify that $\vartheta^{-1}=J \vartheta J$, where $J \in H$ is given by

$$
J=\left(\begin{array}{cc}
-I_{i} & 0 \\
0 & I_{n-i}
\end{array}\right) \text {. }
$$


As a consequence of (4.4) and Lemma 4.2, we note that the convolution of zonal functions (and measures) on $S^{n-1}$ and $\mathrm{Gr}_{i, n}$ is abelian.

Another important ingredient in the proof of Theorem 1.3 are spherical approximate identities. Let $B_{m}(\bar{e})$ be the open geodesic ball of radius $\frac{1}{m}$ at $\bar{e} \in S^{n-1}$, where $m \in \mathbb{N}$ is sufficiently large. A sequence $f_{m}, m \in \mathbb{N}$, of nonnegative $C^{\infty}$ functions on $S^{n-1}$ is called a spherical approximate identity if, for each $m$, the following two conditions are satisfied:

(i) $\int_{S^{n-1}} f_{m}(u) d u=1$;

(ii) $f_{m}(u)=0$ if $u \notin B_{m}(\bar{e})$.

The existence of spherical approximate identities follows from standard techniques similar to the construction of partitions of unity on manifolds (cf. [19, p. 84]). We conclude this section with a well-known auxiliary result, see, e.g., [19, Lemma 2.5].

Lemma 4.3 If $f_{m}, m \in \mathbb{N}$, is a spherical approximate identity, then

(a) $\lim _{m \rightarrow \infty} g * f_{m}=g$ uniformly for every $g \in C\left(S^{n-1}\right)$;

(b) $\lim _{m \rightarrow \infty} \mu * f_{m}=\mu$ weakly for every measure $\mu$ on $S^{n-1}$.

\section{Minkowski valuations}

In this section we collect the background material on translation invariant Minkowski valuations. We also extend the definition of smooth valuations to translation invariant Minkowski valuations which are $\mathrm{O}(n)$ equivariant.

A Minkowski valuation $\Phi: \mathcal{K}^{n} \rightarrow \mathcal{K}^{n}$ is called $\mathrm{O}(n)$ equivariant if for all $K \in \mathcal{K}^{n}$ and every $\vartheta \in \mathrm{O}(n)$,

$$
\Phi(\vartheta K)=\vartheta \Phi K
$$

We denote by MVal the set of continuous translation invariant Minkowski valuations which are $\mathrm{O}(n)$ equivariant and we write $\mathbf{M V a l}_{i}^{(+)}$for its subset of all (even) Minkowski valuations of degree $i$. (This slight abuse of notation should not lead to confusion because in the following all Minkowski valuations will be translation invariant and $\mathrm{O}(n)$ equivariant.) 
Since Minkowski valuations arise naturally, like the projection and the difference operator, from data about projections and sections of convex bodies, they form an integral part of geometric tomography. In the following we give a few well-known examples of Minkowski valuations in MVal (for additional examples, see, e.g., [24, 31, 48]):

\section{Examples:}

(a) For $i \in\{0, \ldots, n\}$, define $\Lambda_{i}: \mathcal{K}^{n} \rightarrow \mathcal{K}^{n}$ by $\Lambda_{i}(K)=V_{i}(K) B$. Clearly, we have $\Lambda_{i} \in \mathbf{M V a l}_{i}^{+}$.

(b) For $i \in\{1, \ldots, n-1\}$, the $i$ th projection operator $\Pi_{i}: \mathcal{K}^{n} \rightarrow \mathcal{K}^{n}$ is an element of $\mathbf{M V a l}_{i}^{+}$.

(c) For $i \in\{1, \ldots, n-1\}$, the (normalized) $i$ th mean section operator $\mathrm{M}_{i} \in \mathbf{M V a l}_{n+1-i}$, introduced by Goodey and Weil [18], is defined by

$$
h\left(\mathrm{M}_{i} K, \cdot\right)=\int_{\mathrm{AGr}_{i, n}} h(K \cap E, \cdot) d \mu_{i}(E)-h\left(\left\{z_{n+1-i}\right\}, \cdot\right) .
$$

Here, $\mathrm{AGr}_{i, n}$ is the affine Grassmannian of $i$-dimensional planes in $\mathbb{R}^{n}$, $\mu_{i}$ is its (suitably normalized) motion invariant measure and $z_{i}(K)$ denotes the $i$ th moment vector of $K$ (see [43, p. 304]).

From Theorem 3.1, one can deduce the following decomposition result for Minkowski valuations (cf. [44, p. 12]):

Lemma 5.1 Suppose that $\Phi \in \mathbf{M V a l}^{(+)}$. Then there are constants $c_{0}, c_{n} \geq 0$ such that for every $K \in \mathcal{K}^{n}$,

$$
h(\Phi K, \cdot)=c_{0}+\sum_{i=1}^{n-1} g_{i}(K, \cdot)+c_{n} V(K),
$$

where the (even) function $g_{i}(K, \cdot) \in C\left(S^{n-1}\right)$ has the following properties:

(a) The map $K \mapsto g_{i}(K, \cdot)$ is a continuous translation invariant (even) valuation of degree $i$.

(b) For every $\vartheta \in \mathrm{O}(n)$ and $K \in \mathcal{K}^{n}$, we have $g_{i}(\vartheta K, u)=g_{i}\left(K, \vartheta^{-1} u\right)$.

It is not known, at this point, whether, for every $K \in \mathcal{K}^{n}$, each function $g_{i}(K, \cdot)$ is the support function of a convex body. Hence, the following important problem is still open: 
Open Problem Suppose that $\Phi \in \mathbf{M V a l}$. Is there a (unique) representation of $\Phi$ of the form

$$
\Phi=\Phi_{0}+\ldots+\Phi_{n}
$$

where $\Phi_{i} \in \mathbf{M V a l}_{i}$ ?

Suppose that $\Phi \in \mathbf{M V a l}$. We define a real-valued translation invariant valuation $\varphi \in$ Val by

$$
\varphi(K)=h(\Phi K, \bar{e}), \quad K \in \mathcal{K}^{n} .
$$

Since $\Phi$ is $\mathrm{O}(n)$ equivariant, we have for $\bar{\eta} \in S^{n-1}$,

$$
h(\Phi K, \bar{\eta})=h(\Phi K, \eta \bar{e})=h\left(\Phi\left(\eta^{-1} K\right), \bar{e}\right)=\varphi\left(\eta^{-1} K\right) .
$$

Consequently, the real-valued valuation $\varphi$ uniquely determines the Minkowski valuation $\Phi$. We call the valuation $\varphi \in$ Val defined by (5.1) the associated real-valued valuation of $\Phi \in \mathbf{M V a l}$.

We can now extend the notion of smooth real-valued valuations to Minkowski valuations in MVal.

Definition $A$ Minkowski valuation $\Phi \in \mathbf{M V a l}$ is called smooth if its associated real-valued valuation $\varphi \in \mathbf{V a l}$ is smooth.

We denote by $\mathbf{M V a l}^{\infty}$ the subset of smooth Minkowski valuations in MVal and we write $\mathbf{M V a l}_{i}^{(+), \infty}$ for the subset of smooth (even) Minkowski valuations in $\mathbf{M V a l}_{i}^{(+)}$.

A description of Minkowski valuations in $\mathbf{M V a l} \mathbf{V}_{1}$ was recently obtained by Kiderlen [24, Theorem 1.3] (extending previous results by Schneider [42]). Here, we state a version of Kiderlen's result for smooth Minkowski valuations:

Theorem 5.2 (Kiderlen [24]) Suppose that $\Phi \in \mathbf{M V a l}_{1}^{\infty}$. Then there exists a unique smooth zonal measure $\mu$ on $S^{n-1}$ such that for every $K \in \mathcal{K}^{n}$,

$$
h(\Phi K, \cdot)=h(K, \cdot) * \mu .
$$

Moreover, the Minkowski valuation $\Phi$ is even if and only if $\mu$ is even.

Since $h(K, u)+h(-K, u)=\operatorname{vol}_{1}(K \mid u)$, we remark that for $\Phi \in \mathbf{M V a l}_{1}^{+, \infty}$, representation (5.3) is equivalent to

$$
h(\Phi K, \cdot)=\operatorname{vol}_{1}(K \mid \cdot) * \mu .
$$


Note that Theorem 5.2 is not a complete characterization of Minkowski valuations in $\mathbf{M V a l}_{1}^{\infty}$ but only a representation result. It is not known which zonal measures on $S^{n-1}$ define a Minkowski valuation by (5.3). However, the following conjecture appears implicitly in [24]:

Conjecture $A \operatorname{map} \Phi: \mathcal{K}^{n} \rightarrow \mathcal{K}^{n}$ is a Minkowski valuation in $\mathbf{M V a l}_{1}$ if and only if there exists a zonal measure $\mu$ on $S^{n-1}$ which is non-negative up to addition of a function $u \mapsto x \cdot u, x \in \mathbb{R}^{n}$, such that for every $K \in \mathcal{K}^{n}$,

$$
h(\Phi K, \cdot)=h(K, \cdot) * \mu .
$$

Recently, the author established a result corresponding to Theorem 5.2 for Minkowski valuations in $\mathbf{M V a l}{ }_{n-1}$ (see [47, Theorem 1.2 \& 1.3]):

Theorem 5.3 Suppose that $\Phi \in \mathbf{M V a l}_{n-1}$. Then there exists a (unique) zonal function $g \in C\left(S^{n-1}\right)$ such that for every $K \in \mathcal{K}^{n}$,

$$
h(\Phi K, \cdot)=S_{n-1}(K, \cdot) * g .
$$

Moreover, the Minkowski valuation $\Phi$ is even if and only if $g=h(L, \cdot)$, for some origin-symmetric body of revolution $L \in \mathcal{K}^{n}$.

It is well known that $\operatorname{vol}_{n-1}(K \mid \cdot)$ for $K \in \mathcal{K}^{n}$ is (up to a constant factor) given by the cosine transform of $S_{n-1}(K, \cdot)$. More precisely,

$$
\operatorname{vol}_{n-1}\left(K \mid u^{\perp}\right)=\frac{1}{2} \int_{S^{n-1}}|u \cdot v| d S_{n-1}(K, v)=\left(S_{n-1}(K, \cdot) * \frac{1}{2}|\bar{e} \cdot .|\right)(u) .
$$

Thus, for $\Phi \in \mathbf{M V a l}_{n-1}^{+, \infty}$, representation (5.4) is equivalent to

$$
h(\Phi K, \cdot)=\operatorname{vol}_{n-1}(K \mid \cdot) * g_{L},
$$

where $g_{L} \in C^{\infty}\left(S^{n-1}\right)$ is the uniquely determined even zonal function such that

$$
h(L, u)=\frac{1}{2} \int_{S^{n-1}}|u \cdot v| g_{L}(v) d v=\left(g_{L} * \frac{1}{2}|\bar{e} \cdot .|\right)(u) .
$$

Here, we have used that the convolution of zonal functions is abelian.

Remark Schneider [42], Kiderlen [24], and the author 47] originally considered translation invariant and $\mathrm{SO}(n)$ equivariant Minkowski valuations of degree 1 and $n-1$, respectively. However, their results imply that for $n \geq 3$, these Minkowski valuations are actually also $\mathrm{O}(n)$ equivariant. 


\section{Proof of the main results}

After these preparations, we are now in a position to give the proof of Theorem 1.1 and the stronger result Theorem 6.3. At the end of this section we prove Theorem 1.2 .

The following result is a refined version of Theorem 1.1 .

Theorem 6.1 Suppose that $\Phi_{i} \in \mathbf{M V a l}_{i}^{+, \infty}, 1 \leq i \leq n-1$. Then there exists a smooth $\mathrm{O}(i) \times \mathrm{O}(n-i)$ invariant measure $\mu$ on $S^{n-1}$ such that for every $K \in \mathcal{K}^{n}$,

$$
h\left(\Phi_{i} K, \cdot\right)=\operatorname{vol}_{i}(K \mid \cdot) * \mu .
$$

The measure $\mu$ can be chosen uniquely from a certain subset $\mathbf{t}_{i}^{\infty}$ of $C^{\infty}\left(S^{n-1}\right)$.

Proof. Let $\varphi_{i} \in$ Val denote the associated real-valued valuation of $\Phi_{i}$. Since $\Phi_{i} \in \mathbf{M V a l}_{i}^{+, \infty}$, we have $\varphi_{i} \in \mathbf{V a l}_{i}^{+, \infty}$. Thus, it follows from Corollary [3.4 that there exists a unique smooth measure $\sigma \in \mathbf{T}_{i}^{\infty}$ such that

$$
\varphi_{i}(K)=\int_{\operatorname{Gr}_{i, n}} \operatorname{vol}_{i}(K \mid E) d \sigma(E), \quad K \in \mathcal{K}^{n} .
$$

By definition (5.1) and the $\mathrm{O}(n)$ equivariance of $\Phi_{i}$, we have for $K \in \mathcal{K}^{n}$ and every $\vartheta \in \mathrm{O}(n-1)$,

$$
\varphi_{i}(\vartheta K)=h\left(\Phi_{i}(\vartheta K), \bar{e}\right)=h\left(\vartheta \Phi_{i} K, \bar{e}\right)=h\left(\Phi_{i} K, \bar{e}\right)=\varphi_{i}(K) .
$$

Therefore, the valuation $\varphi_{i}$ is $\mathrm{O}(n-1)$ invariant. By (6.1), this $\mathrm{O}(n-1)$ invariance carries over to the measure $\sigma$.

We define the set $\mathbf{t}_{i}^{\infty} \subseteq C^{\infty}\left(S^{n-1}\right)$ by

$$
\mathbf{t}_{i}^{\infty}=\left\{\widehat{f}: f \in \mathbf{T}_{i}^{\infty} \mathrm{O}(n-1) \text { invariant }\right\} .
$$

Since for any $\mathrm{O}(n-1)$ invariant $f \in \mathbf{T}_{i}^{\infty}$, we can identify $\widehat{f}$ with an $\mathrm{O}(n-1)$ right invariant and $\mathrm{O}(i) \times \mathrm{O}(n-i)$ left invariant function on $\mathrm{O}(n)$, the set $\mathbf{t}_{i}^{\infty}$ is well defined and consists of $\mathrm{O}(i) \times \mathrm{O}(n-i)$ invariant functions in $C^{\infty}\left(S^{n-1}\right)$.

If we set $\mu=\widehat{\sigma}$, then, by the $\mathrm{O}(n-1)$ invariance of $\sigma$, we have $\mu \in \mathbf{t}_{i}^{\infty}$. Moreover, by (5.2) and (6.1), it follows that for $\bar{\eta} \in S^{n-1}$ and every $K \in \mathcal{K}^{n}$,

$$
h\left(\Phi_{i} K, \bar{\eta}\right)=\varphi_{i}\left(\eta^{-1} K\right)=\int_{\mathrm{Gr}_{i, n}} \operatorname{vol}_{i}(K \mid \eta E) d \sigma(E)=\left(\operatorname{vol}_{i}(K \mid \cdot) * \mu\right)(\bar{\eta}),
$$

which concludes the proof of the theorem. 
We call a measure $\mu$ on $S^{n-1}$ a Crofton measure for the Minkowski valuation $\Phi_{i} \in \mathbf{M V a l}_{i}^{+}$if $h\left(\Phi_{i} K, \cdot\right)=\operatorname{vol}_{i}(K \mid \cdot) * \mu$. In this case, we say $\Phi_{i}$ admits the Crofton measure $\mu$.

\section{Examples:}

(a) For $i \in\{1, \ldots, n-1\}$, we have $\Lambda_{i} \in \mathbf{M V a l}_{i}^{+, \infty}$. By (2.2) and (2.3), the Crofton measure of $\Lambda_{i}$ is a multiple of spherical Lebesgue measure.

(b) For $i \in\{1, \ldots, n-1\}$, it is well known (see, e.g., [18, p. 428]) that the $i$ th projection operator $\Pi_{i} \in \mathbf{M V a l}_{i}^{+}$can be defined by

$$
h\left(\Pi_{i} K, \cdot\right)=\frac{\kappa_{n-1}}{\kappa_{i}} \mathrm{R}_{n-i} \operatorname{vol}_{i}^{\perp}(K \mid \cdot),
$$

where for $f \in C\left(\mathrm{Gr}_{i, n}\right)$, the function $f^{\perp} \in C\left(\mathrm{Gr}_{n-i, n}\right)$ is defined by $f^{\perp}(E)=f\left(E^{\perp}\right), E \in \mathrm{Gr}_{n-i, n}$. Here, $\mathrm{R}_{i}: C\left(\mathrm{Gr}_{i, n}\right) \rightarrow C\left(S^{n-1}\right)$ is the Radon transform defined for $u \in S^{n-1}$ by

$$
\left(\mathrm{R}_{i} f\right)(u)=\int_{u \in E} f(E) d E, \quad E \in \mathrm{Gr}_{i, n} .
$$

Let $\bar{E} \in \mathrm{Gr}_{i, n}$ denote the stabilizer of $\mathrm{O}(i) \times \mathrm{O}(n-i)$. Grinberg and Zhang [19, Lemma 3.2] have shown that

$$
\mathrm{R}_{i} f=f * \mu_{S^{i-1}}
$$

where $\mu_{S^{i-1}}$ is the probability measure on $S^{n-1}$ uniformly concentrated on $S^{i-1}=S^{n-1} \cap \bar{E}$. Consequently, we obtain

$$
h\left(\Pi_{i} K, \cdot\right)=\frac{\kappa_{n-1}}{\kappa_{i}} \operatorname{vol}_{i}(K \mid \cdot) * \mu_{S^{n-i-1}}^{\perp},
$$

where $\widehat{\mu} \widehat{S}^{n-i-1}=\widehat{\mu}_{S^{n-i-1}}^{\perp}$.

(c) For $i \in\{2, \ldots, n-1\}$, the $i$ th mean section operator $\mathrm{M}_{i} \in \mathbf{M V a l}_{n+1-i}$ is not even. However, Goodey and Weil [18, Theorem 5] have shown that (for a suitable constant $c_{n, i}$ )

$$
h\left(\mathrm{M}_{i} K, \cdot\right)+h\left(\mathrm{M}_{i}(-K), \cdot\right)=c_{n, i} \mathrm{R}_{n+1-i} \operatorname{vol}_{n+1-i}(K \mid \cdot) .
$$

Thus, a multiple of $\mu_{S^{n-i}}$ is a Crofton measure for the even part of $\mathrm{M}_{i}$. 
We note that not every Minkowski valuation $\Phi_{i} \in \mathbf{M V a l}_{i}^{+}, 1 \leq i \leq n$, admits a Crofton measure. For example, suppose that $\Phi \in \mathbf{M V a l}_{n-1}^{+}$. Then, by Theorem 5.3, there exists an origin-symmetric body of revolution $L \in \mathcal{K}^{n}$ such that

$$
h(\Phi K, \cdot)=S_{n-1}(K, \cdot) * h(L, \cdot) .
$$

It follows from (5.5) and (5.6) that $\Phi$ admits a Crofton measure if and only if $L$ is a generalized zonoid. However, it is well known that there exist convex bodies (of revolution) which are not generalized zonoids.

An important additional property of Crofton measures of Minkowski valuations is contained in the following result:

Theorem 6.2 Let $\Phi_{i} \in \mathbf{M V a l}_{i}^{+}, 1 \leq i \leq n-1$. If $\mu$ is a Crofton measure for the Minkowski valuation $\Phi_{i}$, then there exists an $\mathrm{O}(i) \times \mathrm{O}(n-i)$ invariant convex body $L \in \mathcal{K}^{n}$ such that

$$
h(L, \cdot)=\widehat{\mathrm{C}_{i} \widehat{\mu}}
$$

Proof. Let $\pi_{1}: \mathrm{O}(n) \rightarrow \mathrm{Gr}_{i, n}$ and $\pi_{2}: \mathrm{O}(n) \rightarrow S^{n-1}$ denote the canonical projections and let $e \in \mathrm{O}(n)$ be the identity map. We denote by $\bar{E}=\pi_{1}(e)$ and $\bar{e}=\pi_{2}(e)$ the stabilizers of $\mathrm{O}(i) \times \mathrm{O}(n-i)$ and $\mathrm{O}(n-1)$, respectively.

Choose an $i$-dimensional subspace $F \in \mathrm{Gr}_{i, n}$ and let $K \subseteq F$ be a convex body. For $u \in S^{n-1}$, a theorem of Hadwiger [23, p. 79] implies that

$$
h\left(\Phi_{i} K, u\right)=f_{i}(F, u) \operatorname{vol}_{i}(K) .
$$

This defines a continuous function $f_{i}: \operatorname{Gr}_{i, n} \times S^{n-1} \rightarrow \mathbb{R}$ satisfying the following properties:

(a) For each $F \in \mathrm{Gr}_{i, n}$, the function $f_{i}(F, \cdot) \in C\left(S^{n-1}\right)$ is the support function of a convex body $L(F)$.

(b) The function $f_{i}(\cdot, \bar{e}) \in C\left(\mathrm{Gr}_{i, n}\right)$ is the Klain function of the associated real-valued valuation $\varphi_{i}$ of $\Phi_{i}$.

(c) For every $\vartheta \in \mathrm{O}(n)$, we have $f_{i}(\vartheta F, u)=f_{i}\left(F, \vartheta^{-1} u\right)$.

Define functions $g_{1} \in C\left(\mathrm{Gr}_{i, n}\right)$ and $g_{2} \in C\left(S^{n-1}\right)$ by

$$
g_{1}(F)=f_{i}(F, \bar{e}), F \in \mathrm{Gr}_{i, n} \quad \text { and } \quad g_{2}(u)=f_{i}(\bar{E}, u), u \in S^{n-1} .
$$


From properties (a) and (c) of $f_{i}$, we deduce that $g_{1}$ is an $\mathrm{O}(n-1)$ invariant function on $\mathrm{Gr}_{i, n}$ and $g_{2}$ is an $\mathrm{O}(i) \times \mathrm{O}(n-i)$ invariant support function of a convex body $L$. Moreover, property (c) of $f_{i}$ implies that for every $\vartheta \in \mathrm{O}(n)$, we have $g_{1}\left(\pi_{1}(\vartheta)\right)=g_{2}\left(\pi_{2}\left(\vartheta^{-1}\right)\right)$. Therefore, we deduce that $g_{1}=\widehat{g_{2}}$.

From property (b) of $f_{i},(\underline{6.1})$ and (3.4), we finally obtain

$$
h(L, \cdot)=h\left(\Phi_{i} K_{\bar{E}}, \cdot\right)=\widehat{g_{1}}=\widehat{\mathrm{K}_{i} \varphi_{i}}=\widehat{\mathrm{C}_{i} \widehat{\mu}}
$$

where $K_{\bar{E}}$ is any convex body in $\bar{E}$ such that $\operatorname{vol}_{i}\left(K_{\bar{E}}\right)=1$.

Suppose that $\Phi_{i} \in \mathbf{M V a l}_{i}^{+}, 1 \leq i \leq n-1$, admits a Crofton measure. Then, by Theorem 6.2, the Klain function of the associated real-valued valuation of $\Phi_{i}$ is essentially the support function of a convex body $L$. In particular, the convex body $L$ determines $\Phi_{i}$ uniquely.

Using Lemma [5.1, we can prove a generalization of Theorem 1.1;

Theorem 6.3 Suppose that $\Phi \in \mathbf{M V a l}^{+, \infty}$. Then there exist constants $c_{0}, c_{n} \geq 0$ and smooth $\mathrm{O}(i) \times \mathrm{O}(n-i)$ invariant measures $\mu_{i}$ on $S^{n-1}$, where $1 \leq i \leq n-1$, such that for every $K \in \mathcal{K}^{n}$,

$$
h(\Phi K, \cdot)=c_{0}+\sum_{i=1}^{n-1} \operatorname{vol}_{i}(K \mid \cdot) * \mu_{i}+c_{n} V(K) .
$$

The measures $\mu_{i}$ can be chosen uniquely from $\mathbf{t}_{i}^{\infty}$.

Proof. Let $\varphi \in \mathbf{V a l}^{+, \infty}$ be the associated real-valued valuation of $\Phi$. By (3.2), there exist smooth valuations $\varphi_{i} \in \mathrm{Val}_{i}^{+, \infty}, 0 \leq i \leq n$, such that $\varphi=\varphi_{0}+\ldots+\varphi_{n}$. By Lemma 5.1 on the other hand, there exist constants $c_{0}, c_{n} \geq 0$ and even $g_{i}(K, \cdot) \in C\left(S^{n-1}\right)$ such that

$$
h(\Phi K, \cdot)=c_{0}+\sum_{i=1}^{n-1} g_{i}(K, \cdot)+c_{n} V(K) .
$$

Clearly, for $i \in\{1, \ldots, n-1\}$, we have $\varphi_{i}(K)=g_{i}(K, \bar{e})$. By Lemma 5.1, $g_{i}(\vartheta K, u)=g_{i}\left(K, \vartheta^{-1} u\right)$ for every $\vartheta \in \mathrm{O}(n)$. Thus, it follows, as in the proof of Theorem 6.1, that there exist unique measures $\mu_{i} \in \mathbf{t}_{i}^{\infty}$ such that

$$
g_{i}(K, \cdot)=\operatorname{vol}_{i}(K \mid \cdot) * \mu_{i} .
$$


We note that the techniques applied in the proof of Theorem 6.1 together with a straightforward adaptation of Lemma 5.1 immediately yield (the proof is almost verbatim the same as the proof of Theorem 6.3) a characterization of valuations with values in the space of continuous functions on $S^{n-1}$ :

Corollary 6.4 A map $f: \mathcal{K}^{n} \rightarrow C\left(S^{n-1}\right)$ is a smooth translation invariant and $\mathrm{O}(n)$ equivariant even valuation if and only if there exist constants $c_{0}, c_{n} \in \mathbb{R}$ and smooth $\mathrm{O}(i) \times \mathrm{O}(n-i)$ invariant measures $\mu_{i}$ on $S^{n-1}$, where $1 \leq i \leq n-1$, such that for every $K \in \mathcal{K}^{n}$,

$$
f(K, \cdot)=c_{0}+\sum_{i=1}^{n-1} \operatorname{vol}_{i}(K \mid \cdot) * \mu_{i}+c_{n} V(K) .
$$

Here, the continuous translation invariant and $\mathrm{O}(n)$ equivariant valuation $f: \mathcal{K}^{n} \rightarrow C\left(S^{n-1}\right)$ is called smooth, if the associated real-valued valuation $\psi \in$ Val, defined by $\psi(K)=f(K, \bar{e}), K \in \mathcal{K}^{n}$, is smooth.

We conclude this section with the proof of Theorem 1.2 .

Theorem 6.5 For every Minkowski valuation $\Phi \in \mathbf{M V a l}^{+}$, there exists a sequence $\Phi^{m} \in \mathbf{M V a l}^{+, \infty}, m \in \mathbb{N}$, such that $\Phi^{m}$ converges to $\Phi$ uniformly on compact subsets of $\mathcal{K}^{n}$.

Proof. Let $f_{m} \in C^{\infty}\left(S^{n-1}\right)$ be a spherical approximate identity. For each $m \in \mathbb{N}$, we define a continuous map $\Phi^{m}: \mathcal{K}^{n} \rightarrow \mathcal{K}^{n}$ by

$$
h\left(\Phi^{m} K, \cdot\right)=h(\Phi K, \cdot) * f_{m}, \quad K \in \mathcal{K}^{n} .
$$

It follows from [24, Proposition 3.2] that the spherical convolution from the right with nonnegative functions and measures maps support functions to support functions. Therefore, $\Phi^{m}$ is well defined. Moreover, it is easy to verify that $\Phi^{m}$ is an even translation invariant Minkowski valuation. By (4.2), $\Phi^{m}$ is also $\mathrm{O}(n)$ equivariant. Consequently, $\Phi^{m} \in \mathrm{MVal}^{+}$.

Let $\varphi^{m} \in \mathrm{Val}^{+}$be the associated real-valued valuation of $\Phi^{m}$ and let

$$
\varphi^{m}=c_{0}^{m}+\varphi_{1}^{m}+\ldots+\varphi_{n-1}^{m}+c_{n}^{m} V
$$

where $c_{0}^{m}, c_{n}^{m} \in \mathbb{R}$ and $\varphi_{i}^{m} \in \mathbf{V a l}_{i}^{+}, 1 \leq i \leq n-1$, be the decomposition of $\varphi^{m}$ into homogeneous parts (which follows from Theorem 3.1). In order to show that for each $m \in \mathbb{N}$, the Minkowski valuation $\Phi^{m}$ is smooth, it suffices to show that $\varphi_{i}^{m}, 1 \leq i \leq n-1$, is smooth. 
Since $f_{m} \in C^{\infty}\left(S^{n-1}\right)$, we deduce from an application of Lemma 5.1 to $\Phi$ and $\Phi^{m}$, that for each $m \in \mathbb{N}$ and every $K \in \mathcal{K}^{n}$, there exist even $g_{i}^{m}(K, \cdot) \in C^{\infty}\left(S^{n-1}\right)$ such that

$$
h\left(\Phi^{m} K, \cdot\right)=c_{0}^{m}+\sum_{i=1}^{n-1} g_{i}^{m}(K, \cdot)+c_{n}^{m} V(K) .
$$

Choose an $i$-dimensional subspace $F \in \mathrm{Gr}_{i, n}$ and let $K \subseteq F$ be a convex body. As in the proof of Theorem 6.2, it follows that for each $m \in \mathbb{N}$, there exists a continuous function $\zeta_{i}^{m}: \mathrm{Gr}_{i, n} \times S^{n-1} \rightarrow \mathbb{R}$ such that

$$
g_{i}^{m}(K, u)=\zeta_{i}^{m}(F, u) \operatorname{vol}_{i}(K \mid F) .
$$

In fact, since $g_{i}^{m}(K, \cdot) \in C^{\infty}\left(S^{n-1}\right)$, we also have $\zeta_{i}^{m}(F, \cdot) \in C^{\infty}\left(S^{n-1}\right)$ for each $F \in \mathrm{Gr}_{i, n}$. Therefore, it follows from the proof of Theorem 6.2 that

$$
\mathrm{K}_{i} \varphi_{i}^{m}=\widehat{\zeta^{m}(\bar{E}, \cdot)}
$$

is smooth. Consequently, $\varphi_{i}^{m}, 1 \leq i \leq n-1$, is smooth which in turn implies that $\Phi^{m} \in \mathrm{MVal}^{+, \infty}$.

It remains to show that $\Phi^{m}$ converges to $\Phi$ uniformly on compact subsets. By (4.8), we may assume that $f_{m}$ is zonal for each $m \in \mathbb{N}$. If $g_{i}(K, \cdot) \in C\left(S^{n-1}\right)$ denotes the degree $i$ component of $h(\Phi K, \cdot)$, then $g_{i}^{m}(K, \cdot)=g_{i}(K, \cdot) * f_{m}$. By Lemma 4.3, $g_{i}^{m}(K, \cdot)$ converges uniformly to $g_{i}(K, \cdot)$ for each $K \in \mathcal{K}^{n}$. Moreover, it is not hard to show that

$$
\left|\varphi_{i}^{m}(K)-\varphi_{i}(K)\right|=\left|\int_{S^{n-1}} g_{i}(K, u) f_{m}(u) d u-g_{i}(K, \bar{e})\right|,
$$

where $\varphi_{i}$ denotes the degree $i$ part of the associated real-valued valuation $\varphi$ of $\Phi$. Hence, the $\varphi_{i}^{m}$ converge to $\varphi_{i}$ pointwise. Since the map $K \mapsto g_{i}(K, \cdot)$ is uniformly continuous on every compact subset of $\mathcal{K}^{n}$ and

$$
\left|\varphi_{i}^{m}(K)-\varphi_{i}^{m}(L)\right| \leq\left\|g_{i}(K, \cdot)-g_{i}(L, \cdot)\right\|_{\infty},
$$

the $\varphi_{i}^{m}$ are equicontinuous on every compact subset of $\mathcal{K}^{n}$. It follows that the $\varphi_{i}^{m}$ converge to $\varphi_{i}$ and thus $\varphi^{m}$ to $\varphi$ uniformly on compact subsets of $\mathcal{K}^{n}$. Since $\mathrm{O}(n)$ is compact, it is easy to verify, using (5.2), that this implies uniform convergence of $\Phi^{m}$ to $\Phi$ on compact subsets of $\mathcal{K}^{n}$. 


\section{A Brunn-Minkowski type inequality}

As an application of Theorem 1.1, we present in this last section the proof of Theorem 1.3. It is based on the techniques developed by Lutwak in [36].

From (2.7) and Theorem 5.2, the author deduced in [47] that for any $\Psi \in \mathbf{M V a l}_{n-1}$, there exist derived Minkowski valuations $\Psi_{i} \in \mathbf{M V a l}_{i}$, where $0 \leq i \leq n-1$, such that for every $K \in \mathcal{K}^{n}$,

$$
\Psi(K+\varepsilon B)=\sum_{i=0}^{n-1} \varepsilon^{n-1-i}\left(\begin{array}{c}
n-1 \\
i
\end{array}\right) \Psi_{i} K
$$

Moreover, the author obtained in [46] an array of geometric inequalities for the intrinsic volumes of derived (non-trivial) Minkowski valuations $\Psi_{i}$. In particular, the following Brunn-Minkowski type inequality was established (cf. [46, Theorem 6.8]): If $K, L \in \mathcal{K}_{\mathrm{o}}^{n}$ and $2 \leq i \leq n-1,1 \leq j \leq n$, then

$$
V_{j}\left(\Psi_{i}(K+L)\right)^{1 / i j} \geq V_{j}\left(\Psi_{i} K\right)^{1 / i j}+V_{j}\left(\Psi_{i} L\right)^{1 / i j}
$$

with equality if and only if $K$ and $L$ are homothetic.

We believe that inequality (7.1) holds in fact for all Minkowski valuations in $\mathbf{M V a l}_{i}$. Theorem 1.3 confirms this conjecture in the case of even valuations and $j=i+1$.

From now on we always assume that $\Phi_{i} \in \mathbf{M V a l}_{i}^{+}$. A critical ingredient in the proof of Theorem 1.3 is the following Lemma.

Lemma 7.1 If $K, L \in \mathcal{K}^{n}$ and $1 \leq i \leq n-1$, then

$$
W_{n-1-i}\left(K, \Phi_{i} L\right)=W_{n-1-i}\left(L, \Phi_{i} K\right)
$$

In particular, there exists a constant $r\left(\Phi_{i}\right) \geq 0$ such that

$$
W_{n-1}\left(\Phi_{i} K\right)=r\left(\Phi_{i}\right) W_{n-i}(K)
$$

Proof: By Theorem 1.2, it suffices to prove the statement for $\Phi_{i} \in \mathbf{M V a l}_{i}^{+, \infty}$. For $K \in \mathcal{K}^{n}$ and $1 \leq i \leq n-1$, we define a measure $s_{i}(K, \cdot)$ on $S^{n-1}$ by

$$
s_{i}(K, \cdot)=\frac{1}{2} S_{i}(K, \cdot)+\frac{1}{2} S_{i}(-K, \cdot) .
$$


Since $\Phi_{i}$ is even, it follows from (2.8) that

$$
W_{n-1-i}\left(K, \Phi_{i} L\right)=\frac{1}{n} \int_{S^{n-1}} h\left(\Phi_{i} L, u\right) d s_{i}(K, u) .
$$

Let $f_{m} \in C^{\infty}\left(S^{n-1}\right)$ be a spherical approximate identity. For each $m \in \mathbb{N}$, we define a function $s_{i}^{m}(K, \cdot) \in C^{\infty}\left(S^{n-1}\right)$ by

$$
s_{i}^{m}(K, \cdot)=s_{i}(K, \cdot) * f_{m} .
$$

From Lemma 4.3, (4.2) and well-known properties of the area measures $S_{i}(K, \cdot)$ (see, e.g., [43, Chapter $\left.4 \& 5\right]$ ), it follows that for each $m \in \mathbb{N}$ :

(a) The map $K \mapsto s_{i}^{m}(K, \cdot)$ is a continuous, translation invariant even valuation of degree $i$.

(b) For every $\vartheta \in \mathrm{O}(n)$ and $K \in \mathcal{K}^{n}$, we have $s_{i}^{m}(\vartheta K, u)=s_{i}^{m}\left(K, \vartheta^{-1} u\right)$.

Moreover, as in the proof of Theorem 6.5, one can show that for each $m \in \mathbb{N}$, the real-valued valuation $K \mapsto s_{i}^{m}(K, \bar{e})$ is smooth. Thus, it follows, as in the proof of Theorem 6.1, that there exist unique measures $\sigma_{i}^{m} \in \mathbf{t}_{i}^{\infty}$ such that

$$
s_{i}^{m}(K, \cdot)=\operatorname{vol}_{i}(K \mid \cdot) * \sigma_{i}^{m} .
$$

Thus, from (7.4), (2.8), Lemma 4.3 and Theorem 1.1, we obtain

$$
W_{n-1-i}\left(K, \Phi_{i} L\right)=\lim _{m} \frac{1}{n}\left\langle\operatorname{vol}_{i}(L \mid \cdot) * \mu_{i}, \operatorname{vol}_{i}(K \mid \cdot) * \sigma_{i}^{m}\right\rangle,
$$

for some $\mu_{i} \in \mathbf{t}_{i}^{\infty}$. From (4.3) and (4.4), it follows that

$$
\left\langle\operatorname{vol}_{i}(L \mid \cdot) * \mu_{i}, \operatorname{vol}_{i}(K \mid \cdot) * \sigma_{i}^{m}\right\rangle=\left\langle\operatorname{vol}_{i}(L \mid \cdot), \operatorname{vol}_{i}(K \mid \cdot) * \widehat{\mu_{i} * \widehat{\sigma_{i}^{m}}}\right\rangle .
$$

Since the measure $\mu_{i}$ is left $\mathrm{O}(i) \times \mathrm{O}(n-i)$ invariant and the measure $\widehat{\sigma_{i}^{m}}$ is right $\mathrm{O}(i) \times \mathrm{O}(n-i)$ invariant, we obtain from Lemma 4.2, that

$$
\widehat{\mu_{i} * \widehat{\sigma_{i}^{m}}}=\mu_{i} * \widehat{\sigma_{i}^{m}}
$$

Combining, (7.5), (7.6) and (4.3), we obtain

$$
W_{n-1-i}\left(K, \Phi_{i} L\right)=\lim _{m} \frac{1}{n}\left\langle\operatorname{vol}_{i}(L \mid \cdot) * \sigma_{i}^{m}, \operatorname{vol}_{i}(K \mid \cdot) * \mu_{i}\right\rangle=W_{n-1-i}\left(L, \Phi_{i} K\right) .
$$


Finally, note that, by the $\mathrm{O}(n)$ equivariance of $\Phi_{i}$, we have $\Phi_{i} B=r\left(\Phi_{i}\right) B$, for some constant $r\left(\Phi_{i}\right) \geq 0$. Thus,

$$
W_{n-1}\left(\Phi_{i} K\right)=W_{n-1-i}\left(B, \Phi_{i} K\right)=W_{n-1-i}\left(K, \Phi_{i} B\right)=r\left(\Phi_{i}\right) W_{n-i}(K) .
$$

By (2.2), our next result is equivalent to Theorem 1.3:

Theorem 7.2 If $K, L \in \mathcal{K}_{\mathrm{o}}^{n}$ and $2 \leq i \leq n-1$, then

$$
W_{n-1-i}\left(\Phi_{i}(K+L)\right)^{1 / i(i+1)} \geq W_{n-1-i}\left(\Phi_{i} K\right)^{1 / i(i+1)}+W_{n-1-i}\left(\Phi_{i} L\right)^{1 / i(i+1)} .
$$

If $\Phi_{i} \mathcal{K}_{\mathrm{o}}^{n} \subseteq \mathcal{K}_{\mathrm{o}}^{n}$, then equality holds if and only if $K$ and $L$ are homothetic.

Proof: By (7.2) and (2.6), we have for $Q \in \mathcal{K}_{\mathrm{o}}^{n}$,

$$
\begin{aligned}
W_{n-1-i}\left(Q, \Phi_{i}(K+L)\right)^{1 / i} & =W_{n-1-i}\left(K+L, \Phi_{i} Q\right)^{1 / i} \\
& \geq W_{n-1-i}\left(K, \Phi_{i} Q\right)^{1 / i}+W_{n-1-i}\left(L, \Phi_{i} Q\right)^{1 / i} \\
& =W_{n-1-i}\left(Q, \Phi_{i} K\right)^{1 / i}+W_{n-1-i}\left(Q, \Phi_{i} L\right)^{1 / i} .
\end{aligned}
$$

From inequality (2.4), we further deduce that

$$
W_{n-1-i}\left(Q, \Phi_{i} K\right)^{i+1} \geq W_{n-1-i}(Q)^{i} W_{n-1-i}\left(\Phi_{i} K\right)
$$

and

$$
W_{n-1-i}\left(Q, \Phi_{i} L\right)^{i+1} \geq W_{n-1-i}(Q)^{i} W_{n-1-i}\left(\Phi_{i} L\right) .
$$

Thus, if we set $Q=\Phi_{i}(K+L)$, we obtain the desired inequality

$$
W_{n-1-i}\left(\Phi_{i}(K+L)\right)^{1 / i(i+1)} \geq W_{n-1-i}\left(\Phi_{i} K\right)^{1 / i(i+1)}+W_{n-1-i}\left(\Phi_{i} L\right)^{1 / i(i+1)} .
$$

Suppose now that equality holds and that $\Phi_{i} \mathcal{K}_{\mathrm{o}}^{n} \subseteq \mathcal{K}_{\mathrm{o}}^{n}$. Since $\Phi_{i} K$ is originsymmetric for every $K \in \mathcal{K}^{n}$, we deduce from the equality conditions of (7.7) and (7.8), that there exist $\lambda_{1}, \lambda_{2}>0$ such that

$$
\Phi_{i} K=\lambda_{1} \Phi_{i}(K+L) \quad \text { and } \quad \Phi_{i} L=\lambda_{2} \Phi_{i}(K+L)
$$

and

$$
\lambda_{1}^{1 / i}+\lambda_{2}^{1 / i}=1
$$


Moreover, since $r\left(\Phi_{i}\right)>0$, (7.3) and (7.9) imply

$$
W_{n-i}(K)=\lambda_{1} W_{n-i}(K+L) \quad \text { and } \quad W_{n-i}(L)=\lambda_{2} W_{n-i}(K+L) .
$$

Hence, we have

$$
W_{n-i}(K+L)^{1 / i}=W_{n-i}(K)^{1 / i}+W_{n-i}(L)^{1 / i}
$$

which implies, by (2.5), that $K$ and $L$ are homothetic.

Acknowledgements. The work of the author was supported by the Austrian Science Fund (FWF), within the project "Valuations on Convex Bodies", Project Number: P 18308.

\section{References}

[1] S. Alesker, Continuous rotation invariant valuations on convex sets, Ann. of Math. (2) 149 (1999), 977-1005.

[2] S. Alesker, Description of translation invariant valuations on convex sets with solution of P. McMullen's conjecture, Geom. Funct. Anal. 11 (2001), 244-272.

[3] S. Alesker, Hard Lefschetz theorem for valuations, complex integral geometry, and unitarily invariant valuations, J. Differential Geom. 63 (2003), 63-95.

[4] S. Alesker, Hard Lefschetz theorem for valuations and related questions of integral geometry, Geometric aspects of functional analysis, 9-20, Lecture Notes in Math., 1850, Springer, Berlin, 2004.

[5] S. Alesker, The multiplicative structure on polynomial valuations, Geom. Funct. Anal. 14 (2004), 1-26.

[6] S. Alesker, Theory of valuations on manifolds. I. Linear spaces, Israel J. Math. 156 (2006), 311-339.

[7] S. Alesker, Theory of valuations on manifolds. II, Adv. Math. 207 (2006), 420-454.

[8] S. Alesker, Theory of valuations on manifolds. IV. New properties of the multiplicative structure, Geometric aspects of functional analysis, 1-44, Lecture Notes in Math., 1910, Springer, Berlin, 2007.

[9] S. Alesker and J. Bernstein, Range characterization of the cosine transform on higher Grassmannians, Adv. Math. 184 (2004), 367-379.

[10] S. Alesker and J.H.G. Fu, Theory of valuations on manifolds. III. Multiplicative structure in the general case, Trans. Amer. Math. Soc. 360 (2008), 1951-1981.

[11] A. Bernig, Valuations with Crofton formula and Finsler geometry, Adv. Math. 210 (2007), 733-753.

[12] A. Bernig, A Hadwiger-type theorem for the special unitary group, Geom. Funct. Anal., in press. 
[13] A. Bernig, A product formula for valuations on manifolds with applications to the integral geometry of the quaternionic line, Comment. Math. Helv., in press.

[14] A. Bernig and L. Bröcker, Valuations on manifolds and Rumin cohomology, J. Differential Geom. 75 (2007), 433-457.

[15] A. Bernig and J.H.G. Fu, Convolution of convex valuations, Geom. Dedicata 123 (2006), 153-169.

[16] W. Casselman, Canonical extensions of Harish-Chandra modules to representations of $G$, Canad. J. Math. 41 (1989), 385-438.

[17] R.J. Gardner, Geometric Tomography, Cambridge University Press, 1995.

[18] P. Goodey and W. Weil, The determination of convex bodies from the mean of random sections, Math. Proc. Camb. Phil. Soc. 112 (1992), 419-430.

[19] E. Grinberg and G. Zhang, Convolutions, transforms, and convex bodies, Proc. London Math. Soc. (3) 78 (1999), 77-115.

[20] J.H.G. Fu, Structure of the unitary valuation algebra, J. Differential Geom. 72 (2006), 509-533.

[21] C. Haberl, Star body valued valuations, Indiana Univ. Math. J., in press.

[22] C. Haberl and M. Ludwig, A characterization of $L_{p}$ intersection bodies, Int. Math. Res. Not. (2006), Article ID 10548, 29 pages.

[23] H. Hadwiger, Vorlesungen über Inhalt, Oberfläche und Isoperimetrie, Springer, Berlin, 1957.

[24] M. Kiderlen, Blaschke- and Minkowski-Endomorphisms of convex bodies, Trans. Amer. Math. Soc. 358 (2006), 5539-5564.

[25] D.A. Klain, An Euler relation for valuations on polytopes, Adv. Math. 147 (1999), $1-34$.

[26] D.A. Klain, Even valuations on convex bodies, Trans. Amer. Math. Soc. 352 (2000), 71-93.

[27] D.A. Klain and G.-C. Rota, Introduction to geometric probability, Cambridge University Press, Cambridge, 1997.

[28] M. Ludwig, Valuations on polytopes containing the origin in their interiors, Adv. Math. 170 (2002), 239-256.

[29] M. Ludwig, Projection bodies and valuations, Adv. Math. 172 (2002), 158-168.

[30] M. Ludwig, Ellipsoids and matrix valued valuations, Duke Math. J. 119 (2003), 159-188.

[31] M. Ludwig, Minkowski valuations, Trans. Amer. Math. Soc. 357 (2005), 4191-4213.

[32] M. Ludwig, Intersection bodies and valuations, Amer. J. Math. 128 (2006), 1409 1428.

[33] M. Ludwig and M. Reitzner, A characterization of affine surface area, Adv. Math. 147 (1999), 138-172.

[34] M. Ludwig and M. Reitzner, A classification of SL(n) invariant valuations, Ann. Math. (2), in press.

[35] E. Lutwak, Mixed projection inequalities, Trans. Amer. Math. Soc. 287 (1985), 91105. 
[36] E. Lutwak, Inequalities for mixed projection bodies, Trans. Amer. Math. Soc. 339 (1993), no. 2, 901-916.

[37] E. Lutwak, D. Yang, and G. Zhang, $L_{p}$ affine isoperimetric inequalities, J. Differential Geom. 56 (2000), 111-132.

[38] E. Lutwak, D. Yang, and G. Zhang, A new ellipsoid associated with convex bodies, Duke Math. J. 104 (2000), 375-390.

[39] E. Lutwak, D. Yang, and G. Zhang, Volume inequalities for subspaces of $L_{p}$ J. Differential Geom. 68 (2004), 159-184.

[40] P. McMullen, Valuations and Euler-type relations on certain classes of convex polytopes, Proc. London Math. Soc. 35 (1977), 113-135.

[41] P. McMullen, Valuations and dissections, Handbook of Convex Geometry, Vol. B (P.M. Gruber and J.M. Wills, eds.), North-Holland, Amsterdam, 1993, pp. 933-990.

[42] R. Schneider, Equivariant endomorphisms of the space of convex bodies, Trans. Amer. Math. Soc. 194 (1974), 53-78.

[43] R. Schneider, Convex Bodies: The Brunn-Minkowski Theory, Cambridge University Press, 1993.

[44] R. Schneider and F.E. Schuster, Rotation equivariant Minkowski valuations, Int. Math. Res. Not. (2006), Article ID 72894, 20 pages.

[45] R. Schneider and W. Weil, Stochastic and Integral Geometry, Springer, Berlin, 2008.

[46] F.E. Schuster, Volume inequalities and additive maps of convex bodies, Mathematika 53 (2006), 211-234.

[47] F.E. Schuster, Convolutions and multiplier transformations, Trans. Amer. Math. Soc. 359 (2007), 5567-5591.

[48] F.E. Schuster, Valuations and Busemann-Petty type problems, Adv. Math. 219 (2008), 344-368.

[49] M. Takeuchi, Modern spherical functions, Translated from the 1975 Japanese original by Toshinobu Nagura. Translations of Mathematical Monographs 135, American Mathematical Society, Providence, RI, 1994.

[50] N.R. Wallach, Real reductive groups. I, Pure and Applied Mathematics 132, Academic Press, Inc., Boston, MA, 1988.

Vienna University of Technology

Institute of Discrete Mathematics and Geometry

Wiedner Hauptstraße 8-10/1046

A-1040 Vienna, Austria

franz.schuster@tuwien.ac.at 